University of Nebraska - Lincoln

DigitalCommons@University of Nebraska - Lincoln

Sociology Department, Faculty Publications

Sociology, Department of

6-2009

\title{
Too Many Friends: Social Integration, Network Cohesion and Adolescent Depressive Symptoms
}

Christina D. Falci

University of Nebraska-Lincoln, cfalci2@unl.edu

Clea McNeely

University of Tennessee - Knoxville, cmcneely@utk.edu

Follow this and additional works at: https://digitalcommons.unl.edu/sociologyfacpub

Part of the Sociology Commons

Falci, Christina D. and McNeely, Clea, "Too Many Friends: Social Integration, Network Cohesion and Adolescent Depressive Symptoms" (2009). Sociology Department, Faculty Publications. 185.

https://digitalcommons.unl.edu/sociologyfacpub/185

This Article is brought to you for free and open access by the Sociology, Department of at DigitalCommons@University of Nebraska - Lincoln. It has been accepted for inclusion in Sociology Department, Faculty Publications by an authorized administrator of DigitalCommons@University of Nebraska - Lincoln. 


\title{
Too many friends: Social Integration, Network Cohesion and Adolescent Depressive Symptoms
}

\author{
Christina Falci, University of Nebraska-Lincoln \\ Clea McNeely, University of Tennessee-Knoxville
}

Using a nationally representative sample of adolescents, we examine associations among social integration (network size), network cohesion (alter-density), perceptions of social relationships (e.g., social support) and adolescent depressive symptoms. We find that adolescents with either too large or too small a network have higher levels of depressive symptoms. Among girls, however, the ill effects of over-integration only occur at low levels of network cohesion. For boys, in contrast, the ill effects of over-integration only occur at high levels of network cohesion. Large social networks tend not to compromise positive perceptions of friend support or belonging; whereas, small networks are associated with low perceptions of friend support and belonging. Hence, perceptions of social relationships mediate the ill effects of under-integration, but not over-integration, on depressive symptoms.

Roughly 30 percent of adolescents report moderate to severe depressive symptoms (Rushton, Forcier and Schectman 2002). The early occurrence of depression in adolescence sets a foundation for recurrent and severe depressive episodes later in life (Belsher and Costello 1988; Kovacs et al. 1984). Depression in adolescence is also an urgent health concern. Depressive symptoms are the strongest predictor of suicidal ideation which, in turn, predicts suicide attempts (Kandel, Raveis and Davies 1991). Suicide is the fourth leading cause of death among 10-14 year olds in the United States and the third leading cause of death among 15-24 year olds (Anderson 2001). This research explores how the network structure and perception of adolescent friendships influence depressive symptoms in adolescence.

Several decades of research make a clear link between social relationships and depressive symptoms in adolescence. This is not surprising given that cultivating and managing peer relationships is a central developmental task of adolescence, requiring much time and energy. The vast majority of research on peer relationships focuses on perceptions

The authors gratefully acknowledge funding from the William T. Grant Foundation. We would also like to express thanks to Jim Moody for graciously sharing his SAS Programs for Analyzing Networks users manual. Direct correspondence to Christina Falci, University of Nebraska-Lincoln, Department of Sociology, 711Oldfather Hall, Lincoln, NE 68588-0324. E-mail: cfalci2@unl.edu. 
of those relationships. Adolescents who perceive high levels of affection and acceptance from peers report fewer depressive symptoms (Beam et al. 2002; Formoso, Gonzales and Aiken 2000; Furman and Buhrmester 1992). Relatively few studies investigate how the structure of social networks - the pattern of ties between members of a social network relate to depressive symptoms among adolescents (for exceptions see Hansell 1985; Ueno 2005). The focus of social network analysis is the ties between individuals rather than individuals' experiences or perceptions of relationships (Wasserman and Faust 1994). An advantage of network structure analysis is the ability to go beyond individual perceptions: this research does not rely solely on self-reports from adolescents.

The choice to rely solely on adolescent self-report is often due to methodological challenges: reports from adolescents' friends are costly to collect and seldom exist in secondary datasets. However, studies that rely only on self-report of friendships, especially as they are linked to mental health, suffer from two significant limitations. First, self-reported perceptions, including how many friends one has and how supportive those friendships are, may be influenced by current or previous experiences of depressed mood (Turner and Turner 1999). Second, and more broadly, by relying on adolescents' self-report of their friendship experiences we fail to understand the influences of the structural properties of their friendship network. Structural network characteristics cannot be accurately measured from the perceptions of a single member of the network (Wellman 1988). In this article, social network theory and methods are applied to investigate the influence of network structure on adolescent depressive symptoms, focusing on two dimensions of network structure, social integration and network cohesion.

Social integration is the aspect of network structure that has received the most study. Broadly defined, it is the degree to which an individual is connected to other individuals in a network. Social integration has three dimensions: the number of social ties, the type of tie (e.g., close friend vs. acquaintance) and the frequency of contact (House, Umberson and Landis 1988). Of these three dimensions, the number of social ties, or the size of an adolescent's friendship network, has received the most empirical attention in research on adolescents (Ennett et al. 2006; Ueno 2005). Social integration is hypothesized to have a curvilinear relationship with depressive symptoms such that having either too few friends (under-integration) or too many (over-integration) is harmful to mental health (Durkheim 1951; Pescosolido and Levy 2002). Although empirical research supports the claim that adolescents with too few friendship ties are more likely to experience depressive symptoms (Brendgan, Vitaro and Bukowski 2000; Ueno 2005), the possibility that having too many friends might be linked to depressive symptoms in adolescents has not been adequately explored. ${ }^{1}$ 
The possibility that properties of social network structure function multiplicatively rather than additively has seldom been explored for adolescent networks (for an exception see Haynie 2001). Previous research on adolescents typically has treated different dimensions of network structure as theoretically independent constructs (Ennett et al. 2006; Ueno 2005). This research tests whether the association between social integration and depressive symptoms varies as a function of the cohesiveness of the friendship network. Network cohesion refers to the degree of interconnections within a social network. Within a network of friendship ties, network cohesion assesses the extent to which an adolescent's friends are friends with one another. This research also explores how the association between the two dimensions of network structure - social integration and network cohesion - affect depressive symptoms differently for boys and girls. Previous research has not assessed gender variation in the association between network structure and depressive symptoms. Yet, social network research in organizational settings suggests the effect of network structure on worker outcomes differs by gender (Burt 1998; Ibarra 1997). Similar patterns might occur when investigating the network structure of adolescent friendships on depressive symptoms.

In addition to making three new contributions to research on how adolescent friendship networks affect mental health - testing for a curvilinear relationship between social integration and depressive symptoms, testing whether network cohesion modifies that association and testing for gender differences - this article extends and verifies the findings of Ueno's (2005) foundational research linking small network size to depressive symptoms. Like Ueno, the argument that the presence of a single close friend is more important to an individual's well-being than the number of friends is investigated (Baumeister and Leary 1995). Ueno found that a single close friend is not sufficient to protect against depressive symptoms. The current research extends this line of inquiry by taking into account reciprocity. Ueno (2005) also found that the influence of under-integration on depressive symptoms is mediated by perceived belonging. The current research explores an additional mediator, perceived support from friends, and also tests whether adolescents' perceptions of belonging and support can mediate the effects of over-integration, as well as under-integration, on depressive symptoms.

\section{Under-Integration and Depressive Symptoms}

Adolescents who are under-integrated (i.e., they have very few or no friends) are at greater risk for depressive symptoms (Brendgen et al. 2000; Ueno 2005). Adolescents seek social connection with peers (Baumeister and 
Leary 1995; Chu 2005). If their efforts go unfulfilled, they are more likely to experience exclusion and loneliness, and to develop depressive symptoms (Rosenberg and Cullough 1981). Because these findings hold for both boys and girls, we do not expect the effect of under-integration to vary by gender. We also do not expect the effect of under-integration to vary by network cohesion. By definition, socially isolated adolescents cannot have cohesive networks. It is necessary to have a minimum of two friends to know the extent to which one's friends are friends with one another. Even among adolescents with more than two friends, but relatively small networks, we do not expect the effect of network size to vary by network cohesion.

\section{Is One Friend Enough?}

Some argue that a single close friend can provide sufficient intimacy, support and companionship for an adolescent's well-being (Baumeister and Leary 1995). We hypothesize that small network size contributes to depressed mood even when an adolescent has a reciprocated close friendship. Essentially, we do not expect one close friend to meet all of an adolescent's needs for social connection. A single friend does not give access to social status or information channels, both of which help a student fit in at school (Crosnoe and McNeely in press; Walker, Wasserman and Wellman 1993). There is previous empirical support for this hypothesis (Ueno 2005).

\section{Meditation of Under-Integration by Perceptions of Belonging and Support from Friends}

We examine perceptions of belonging and support from friends as two mediating mechanisms by which under-integration might lead to higher levels of depressive symptoms among adolescents (House et al. 1988; Ueno 2005). Perceived belonging at school is the sense of being a part of the social fabric at school, of fitting in. Not having friends to sit with in the lunch room or to pass notes to in class can undermine feelings of belonging. Seemingly innocuous moments, such as passing time or choosing teams for a class project, become laden with the potential for feelings of rejection and isolation. Thus, adolescents with few friends are less likely to feel that they belong at school. Perceived support from friends is the extent to which adolescents believe that their friends care about them. Adolescents with few friends might perceive less support than adolescents with more friends. In light of these predictions, we expect that perceived belonging and friend support will mediate the relationship between small network size and depressive symptoms in adolescence. Previous research has demonstrated that support from friends and a sense of belonging are inversely related with depressive symptoms (Laible, Carlo and Farraelli 2000; McNeely and Falci 2004). Furthermore, using the same Add Health 
data set, Ueno (2005) found that a sense of belonging mediated the relationship between network size and adolescent depressive symptoms when network size was modeled as a linear relationship. Support from friends has not been explored previously as a mediator.

\section{Over-Integration and Depressive Symptoms}

Over-integration is often theorized to result in greater mental health problems (Pescosolido and Levy 2002). Durkheim (1951) argued that over-integration could lead to altruistic suicide, where a person takes his own life "because it is his duty." (Durkheim 1951:219) In this instance, an individual sacrifices himself for his community (e.g., a soldier jumping on a live grenade to save fellow soldiers or an elderly person in poor health ending his life so as not to burden loved ones). Our research investigates depressive symptoms, not suicide, but the idea of duty or obligation is partly why over-integration may lead to higher levels of depressive symptoms. The role of friendship entails a set of behavioral expectations, such as providing comfort or assistance and spending time together. As the number of friends an individual has increases, the time and energy costs of maintaining them also increases and may outweigh the benefits of having friends (Eder 1985; Eder, Evans and Parker 1995). Having obligations to many friends may leave a person feeling worn out. Too many friends could result in role strain because the demands on the adolescent to fulfill the role of friendship are greater than his or her ability to enact the role (Pearlin 1983). Role strain, in turn, can lead to poor self-assessment of one's success in enacting the friendship role. Both role strain and negative role performance evaluations are likely to lead to depressive symptoms (Thoits 1991).

\section{Does the Effect of Over-Integration Vary by Network Cohesion and Gender?}

Previous research has focused on the independent effects of distinct network characteristics; however, the negative effect of having too many friends may depend on levels of network cohesion. Network cohesion can be represented as a continuum from low to high cohesion. At one extreme, an adolescent might have a completely fragmented local network where none of the adolescent's friends are friends with one another. At the other extreme, an adolescent could have a closed network, where all of the adolescent's friends nominate each other as a friend. Adolescents tend to fall somewhere in the middle of these two extremes, but the former extreme is more common than the latter (see appendix A).

One can understand the import of the cohesiveness of network structure intuitively by looking at friendship structure visually. Figure 1 shows two large friendship networks of equal size (15 actors), but with varying levels of network cohesion. The network in Panel A has low 
Figure 1. Alter Networks with Varying Levels of Alter-Density

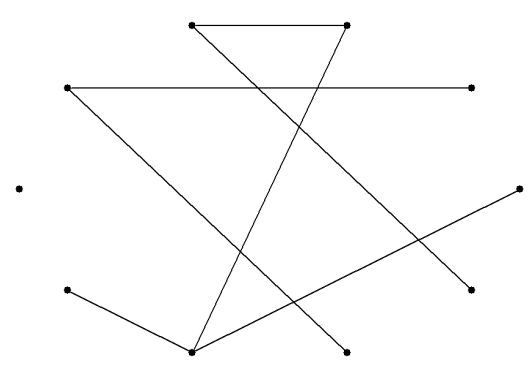

Panel A

Alter-density $=15 \%$

Large Fragmented Network

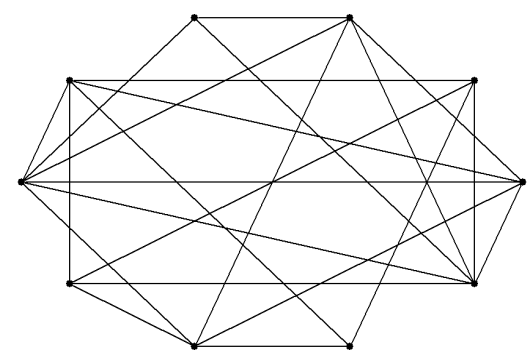

Panel B

Alter-density $=50 \%$ Large Cohesive Network

network cohesion; referred to as a large fragmented network. Few of the friends within this adolescent's friendship network are friends with each other. Of all the possible ties among alters (i.e., the adolescent's friends), just 15 percent are actual friendship ties. The network in Panel B has high network cohesion; referred to as a large cohesive network. In this network, an adolescent's friends also tend to be friends with one another. Fifty percent of the possible ties between the adolescent's friends are present. Experiences with peer relationships may dramatically differ between these two network structures leading to differences in depressive symptoms.

Specifically, adolescents who have large fragmented networks may report higher levels of depressive symptoms than adolescents with large cohesive networks. Large fragmented networks should exacerbate the role strain of numerous friendships. Because an adolescent's friends do not know one another in a fragmented network - at least not very well - any given friend will be unaware of the various demands other friends might place on the adolescent. Consequently, the social costs and obligations of having numerous friends will be greater in fragmented networks and the adolescent may experience greater role strain. In contrast, when large networks are cohesive, the cohesion might provide some protection from the potential costs of many friendships. Large cohesive networks should be better able to share and coordinate social support to a network member, thereby preventing the overburdening of any one network member. Furthermore, knowing that other friends are supporting a friend in need might alleviate feeling inadequate about one's own role performance. Thus, large cohesive networks might buffer the negative effects of over-integration on depressive symptoms.

There may, however, be gender variation in the patterns hypothesized above. Similar network structures, such as a large cohesive friendship 
networks, can have different effects on health outcomes if the nature of social interactions occurring within those similar network configurations differ (Friedkin 2004). Patterns of social interaction within an adolescent's friendship network are likely to vary by the adolescent's gender for several reasons. First, adolescent boys face greater pressure to conform to masculine roles than girls do for feminine roles (Zucker,Wilson-Smith and Stern 1995; Fagot 1985) and a failure to conform to norms of masculinity can result in ridicule from friends (Messerschmidt 2000; Chu 2005). Second, adolescent boys' social interactions tend to revolve around social activities, whereas adolescent girls are more likely to report engaging in mutually supportive interactions with friends (Nada-Raja, McGee and Stanton 1992; Frydenberg and Lewis 1993). Third, girls are more likely than boys to privilege the needs of others over their own needs (Rosenfield, Lennon and White 2005). Clearly, adolescent boys and girls tend to approach or experience peer friendships in a different manner.

These potential gender differences in patterns of social interactions with friends may lead to gender differences in the effect of network structure on depressive symptoms. Specifically, high levels of social cohesion may not be as beneficial for adolescent boys compared to adolescent girls. Highly cohesive networks will be able to exert more pressure on boys to conform to group masculinity norms than less cohesive networks (Friedkin 1984; Haynie 2001; Eder and Enke 1991). In order to avoid ridicule from nonconformity, an adolescent boy will act in a manner consistent with norms, even if those norms do not represent him personally (Chu 2005). Inauthentic self-presentations are likely to lead to poor mental health outcomes (Gecas 1986). Since adolescent boys with highly cohesive networks may be more inclined to have inauthentic self-presentations than boys in less cohesive networks, they may also be more likely to have higher levels of depressive symptoms. As a result, high levels of social cohesion may not buffer the effect of over-integration on depressive symptoms for boys.

Second, large fragmented networks will be particularly detrimental for adolescent girls. Adolescent girls are likely to have higher levels of identity salience to the role of friendship than boys. Adolescent girls report a higher number of peer-related stressors than boys (Green 1988), and previous research on adults finds that both women and men report receiving more support from friendships with women than friendships with men (House et. al. 1988). When role identities, such as friendships, have high salience, the ill effects of role strain related to the role should be exacerbated (Marcussen, Ritter and Safron 2004; Thoits 1991). Peer-related stressors appear to have a stronger influence on girls' mental health than boys' (Joyner and Urdy 2000; Marcotte, Alain and Gosselin 1999). For girls, then, large fragmented networks are likely to be especially bad. In sum, highly cohesive networks will buffer the negative effects of over-integration on depressive symptoms 
for girls but not boys, and highly fragmented networks will exacerbate the negative effects of over-integration for girls relative to boys.

\section{Mediation of Over-Integration by Social Support and Social Belonging}

Previous research consistently documents two linear relationships: (1. as network size increases, so do adolescents' perceptions of belonging and support (Haines, Beggs and Hurlbert 2002; Walker et al. 1993); and (2. as perceptions of belonging and support increase, depressive symptoms decrease (Laible et al. 2000; McNeely and Falci 2004). If this is the case, then perceptions of belonging and support cannot mediate the hypothesized association between over-integration and depressive symptoms. Perceptions of belonging and support can only mediate the ill effects of over-integration if over-integration leads to lower levels of belonging and support. Although we predict positive linear relationships between network size and perceptions of belonging and support, we test the competing hypothesis that over-integration compromises perceived peer support and the sense of belonging.

\section{The Current Study}

Our research assesses for the first time the possibility of a curvilinear relationship between social integration and depressive symptoms in adolescence. Both under-integrated and over-integrated adolescents are hypothesized to report higher levels of depressive symptoms than adolescents with average-sized social networks. However, the effect of over-integration on depressive symptoms will vary as a function of both network cohesion and gender. For girls, large networks will not compromise well-being if they are cohesive. For boys, however, network cohesion will not protect against the negative effects of over-integration. Finally, perceptions of friend support and belonging will mediate the association between under-integration and depressive symptoms, but not the association between over-integration and depressive symptoms. Higher levels of depressive symptoms among over-integrated adolescents probably result from higher levels of role strain, although it is not possible to test this potential mechanism with the data used in this study.

\section{Methods}

\section{Sample}

Add Health is a stratified sample of 132 junior and senior high schools in the United Sates (Udry 2003). An in-school survey was administered in 1994. All social network measures are created from friendships nominations 
collected in the in-school questionnaire. All students present on the day of the survey were asked to list up to ten friends, five of each gender. Students could nominate friends in or outside of their school (of course, network measures can only be constructed when the sender and receiver attended the same school). Schools provide a good approximation of peer social networks in adolescence, because the majority of friendship ties in adolescence occur within school. Within this study, 68 percent of all friendship nominations were sent to a friend at school. For this analysis, we excluded 27 of the 132 schools for the following reasons: administrator refusal to collect network data $(n=8)$, data processing errors $(n=1)$, all students at the school were enrolled in special education $(n=2)$ and response rates were less than 70 percent, creating excessive missing data in the friendship nominations. We selected the cutoff of 70 percent based on the recommendation from recent research on non-response in social networks (Kossinets 2006).

Approximately one year after the in-school survey, an in-home interview was conducted with a nationally representative sub-sample of 20,747 students who were on the school rosters or had been interviewed in school. At that same time, a survey was given to a parent, in most cases the mother. All non-network measures in this study are drawn from the in-home surveys of the adolescent and parent. The analytic sample for this research is restricted to adolescents who completed both the in-home and in-school questionnaire. Numerous adolescents did not fill out both the in-home and in-school survey, because adolescents who did not fill out the in-school survey were targeted for the in-home survey $(n=5,391)$. Adolescents who attended a school excluded from this study, as described above $(n=1,267)$, and who were not on school rosters because they did not receive a preassigned ID number $(n=437)$ are dropped. A pre-assigned ID number is necessary for creating network linkages among actors within the school friendship network. Furthermore, a friendship nomination sent to a student without a pre-assigned ID number or received from a student without a pre-assigned ID is a missing friendship nomination. Adolescents who had personal networks in which more than 30 percent of their friendship nominations were missing are also dropped $(n=1,257)$.

The sample is restricted to white, black and Latino adolescents who did not report having a same-sex romantic attraction. Excluding 1,605 racial minority students increased the likelihood that most students in the sample would attend schools with other students of the same race. Because adolescent friendship networks are highly segregated by race (Moody 2001a), a lack of racial representation within one's school may affect the size and density of an adolescent's friendship network within school and possibly on the effects of network structure. It is beyond the scope of this research to investigate these unique circumstances. 
Adolescents who self-identified as having a same-sex romantic attraction $(n=665)$ are also excluded because we did not want to conflate the close friend network variables with a romantic relationship. Finally, cases were lost because they did not have a valid sampling weight $(n=759)$ or had missing data from the in-home questionnaire $(n=269)$. The final size of the analytic sample is 9,097.

Although Add Health has a good demographic representation of adolescents in the United States, the total number of friendship nominations among actors in a school is underreported within this data set. The distribution of friendship nominations is truncated because adolescents were only able to nominate five same-gender friends. Friendship ties occur more often within - rather than across - gender (Moody 2001b). For the sample used in this study, 69 percent of girls used all five female friend nominations and 56 percent of boys used all five male friend nominations. These percentages include school and non-school friend nominations. Importantly, nominating friends outside of school precluded adolescents from nominating friends within school. Because the number of friendship nominations is underreported, the estimated size of an adolescents' local network will be lower than the true population mean (Kossinets 2006). Since our measure of network size is truncated we expect our findings on the influence of overintegration on depressive symptoms to be lower bound estimates. In other words, due to data limitations our findings will represent a conservative estimate of the effect of over-integration on depressive symptoms.

\section{Measures}

Depressive symptoms are measured with a 15-item modified CES-D scale. Consistent with previous research, we excluded the four items within the interpersonal symptoms sub-scale of the CES-D (e.g., questions about feeling lonely) because they are closely related to the independent variables in this study. The modified version of the CES-D has a range between 1 and 45 with good reliability as measured by Cronbach's alpha ( $\alpha$ $=.82$ ). Perceived belonging is a three-item scale developed by Bollen and Hoyle (1990). Students were asked how much they agreed or disagreed with the following statements: you feel like you are a part of your school, you are happy to be at your school and you feel close to people at school. Response categories ranged from 1, representing "strongly disagree" to 5 , representing "strongly agree." A confirmatory factor model fits the data well in this sample (McNeely 2005). The perceived belonging measure ranges between 1 and 13 and has good reliability for a three-item scale ( $\alpha$ $=.78$ ). Perceived friend support is measured by a single question asking the adolescent to indicate how much they think their friends care about them. The response choices ranged from 1 "not at all" to 5 "very much." 
All social network measures are derived from an adolescent's local network, defined as one focal actor (ego) and the actor's direct contacts (alters). All nominated friends who do not attend the same school have to be excluded from the local network so the network represents friendships within the school only. Social integration is operationalized by network size. Network size is a count of the number of alters who make or receive a friendship nomination from the focal adolescent, plus the adolescent him- or herself. Alters are counted only once, regardless of whether the friendship nomination is reciprocated. Wasserman and Faust (1994) call this measure degree, defined as the union of alters who send and/or receive a school friendship nomination to or from ego, plus ego. We use the term network size because it is more intuitive. Theoretically, network size can range from 1, indicating that the adolescent has neither sent nor received any friendship nominations, to the total number of students in the respondent's school minus one. In practice, network size is limited by the fact that each adolescent is only allowed to nominate five friends of each gender.

Network cohesion is operationalized by alter-density, which assesses the extent to which the alters in a local network are friends with one another. It is calculated by dividing the actual number of friendship ties between an adolescent's alters (i.e., friends) by the total number of possible ties, excluding in both the denominator and numerator ties with the focal adolescent. The alter matrix was symmetrized, prior to calculating alter-density, to correct for the potentially missing nominations due to the right censoring of friendship nominations (Kossinets 2006). We use alter-density as opposed to ego-density because we do not want to conflate the extent to which a respondent's friends know one another with the respondents' level of friendship reciprocity (i.e. the extent to which a friend nominated by the respondent also nominates the respondent as a friend). It is impossible to calculate the alterdensity of a local friendship network that does not have at least two alters. Adolescents with 1 or no alters in their school network are assigned the value of zero for alter-density. Alter-density ranges from 0 , indicating none of the focal adolescent's friends are friends with each other, to 1, indicating that all of the focal adolescent's friends are friends with each other.

The measure of a reciprocated close friendship is developed to test whether having a single close friend is sufficient to prevent elevated depressive symptoms among adolescents with few friends. Although we do not have data on the closeness of each nominated friendship, we can take advantage of the order of friendship reporting, gender of the friendship and whether the friendship is reciprocated to ensure that a modicum of closeness is present within the friendship. Respondents were asked to nominate their closest male and female friend first. The first nomination could be a romantic relationship or a best friend. To avoid confusion with romantic relationships, we focus on same-gender friendships and 
exclude adolescents who report a same-sex romantic attraction from the analytic sample. For this analysis, an adolescent has a reciprocated close friendship if the first friend they list of the same gender attends their same school and reciprocates the friendship by nominating the adolescent as a friend. Unreciprocated close friendship indicates that the first same-sex friend nominated by the adolescent attends the same school but does not reciprocate the friendship nomination. Non-school close friendship indicates the first same-sex nominated friend did not attend the same school. It is impossible to determine if the non-school friend reciprocated. The omitted reference category for these close friendship variables is the adolescents who reported no close friend of the same gender.

Two additional friendship network measures are included as controls. First, the number of nominations made to friends who do not attend the school. On average, adolescents nominated two friends who did not attend his or her school. With this control measure, the coefficient for network size can be interpreted as the independent effect of a student's network size at school. In multivariate analyses, this variable is mean-centered because it interacts with gender. Second, the number of missing school friendship nominations (i.e., friendship nominations sent to or received from students who were not on the school roster and hence did not have a pre-assigned ID). More than 80 percent of the adolescents in the sample neither nominate nor receive nominations from students without ID numbers, and an additional 15 percent are missing just one school friendship nomination.

The following sociodemographic characteristics are included in multivariate models as potential confounders because they are associated with mental health outcomes and network structure (Eccles et al. 1993; Moody 2001a; Gifford-Smith and Brownell 2003): gender, grade in school, race/ethnicity, household income, school size, residential mobility and length of time in current school. Table 1 reports the weighted descriptive statistics for these variables. Household income is based on parental report of income on the parent survey. Missing values for this variable were replaced with the sample mean ( $n=2,072,23$ percent of the sample) and household income is logged in all multivariate analyses. School size indicates the number of students attending the respondent's school. Residential mobility indicates the number of years the adolescent has lived at his or her current residence. Length of time in current school indicates the number of years the adolescent has attended his or her current school.

\section{Analytic Strategy}

The social network measures were created using PROC IML procedures in SAS 9.1. OLS regression models test hypotheses regarding the curvilinear relationship between social integration and depressive symptoms. All 
analyses are run in SAS 9.1 and adjusted for Add Health's complex sampling design (Chantala 2006). Specifically, all analyses are weighted to adjust for over-samples and nonresponse, and the standard errors are adjusted to take into account the stratified sampling plan and the clustering of students within schools.

The joint test of curvilinear relationships and interaction effects for degree, alter-density and gender required testing a four-way interaction. Inclusion of multiple interaction terms poses the potential problem of multicollinearity. Several approaches explore potential multicollinearity problems. First, network size is transformed to Z-scores and alter-density is mean-centered (Jaccard, Wan and Turrisi 1990). The VIFs for the interaction terms in the four-way model ranged $2-5$, which are high but below the acceptable threshold of 10 (Hair et al. 2006). Second, because of the presence of multicollinearity, the stability of the beta coefficients is assessed by running the four-way interaction model on randomly selected sets of half of the analytic sample (Echambadi and Hess 2007). These subset analyses replicated our results. Third, only interaction terms that explained additional variance in the model, using an F-test, are kept (Kromrey and Foster-Johnson 1998). Finally, additional analyses stratified by gender and levels of alter-density verified the results of the interactions.

Applying ordinary least squares regression to a skewed dependent variable raises the concern of possible spurious interaction terms(Osgood, Finken and McMorris 2002; Haynie and Osgood 2005). For this reason, the CESD is transformed using IRT methods (the graded response model; Samejima 1969) in Mplus. Then, all analyses were duplicated using the transformed CESD (i.e., factor scores from the Mplus confirmatory factor analysis) in a Tobit regression model with the IVE-ware SAS module to adjust for the complex survey design (Raghunathan, Solenberger and Van Hoewyk 2002). The results did not differ in significance or effect size. For simplicity and interpretability, the results from the OLS regression are presented. Finally, to investigate the possibility that unexplained variation in individual outcomes might be due to unspecified differences between schools random effects models were estimated in Stata 9 using the xtreg command. Again, the results did not differ in any substantive way from the results obtained using the SAS surveyreg procedures. To do an additional check of school size (range $=26$ to more than 3,000 students), analyses were run on a sample with a minimum school size of 500 students, and the results did not change.

\section{Results}

\section{Network Descriptive Statistics}

Table 1 reports weighted descriptive statistics. On average, adolescents nominated or received nominations from almost eight friends in school 
and nominated about two friends outside of school. The largest friendship network consisted of 34 adolescents. The average alter-density is 21 percent. In other words, roughly 20 percent of an adolescent's friends nominate one another as friends. Although the maximum reaches 100 percent for alter-density, few adolescents - just 2 percent of the sample - reach this level of alter-density. Although alter-density is fairly skewed, there is good variation in network size across all levels of alter-density (see Appendix A). The vast majority of the sample, 88 percent, identified at least one same-sex friend. Forty-nine percent of those who nominated a same-sex friend (45 percent of the full sample) had that friendship reciprocated (i.e., that student also nominated the focal adolescent as a friend). The remaining adolescents either made a nomination that could not be reciprocated due to the study methodology (e.g., the student did not attend the school) or was not reciprocated for some other reason (e.g., the alter did not perceive the focal adolescent to be a friend).

\section{Network Structure and Depressive Symptoms}

A primary hypothesis of this study is that having too few or too many friends is associated with greater depressive symptoms. The multivariate models testing this hypothesis are presented in Table 2. All models control for the number of friends who do not attend the school, the number of friends missing from the network, and the following demographic characteristics: grade, gender, race, household income, school size, number of years at current school and number of years at current residence. As expected, Model 1 reveals a curvilinear relationship between network size and depressive symptoms; adolescents with very small and very large networks report slightly higher levels of depressive symptoms. The squared term for network size is statistically significant and contributes additional variation ( $F=20.03, p<.001$ ). As network size increases, depressive symptoms decline until network size reaches approximately 12 friends. Beyond 12 friends, the direction of the association reverses, and depressive symptoms increase along with network size. Adolescents with a network of 24 friends experience, on average, the same level of depressive symptoms as adolescents with no friends.

All models in Table 2 also show an intriguing association between the number of non-school friends and depressive symptoms, which we verified in analyses stratified by gender. For boys, there is no association between the number of nominated friends who do not attend the school and depressive symptoms ( $b=-.042, n s)$. For girls, the number of friends nominated outside the school is positively associated with depressive symptoms (Model 1: $-.042+.223=.181, \mathrm{p}<.05$ ). Since we do not know any characteristics of these friends outside of school, which 
Table 1: Weighted Descriptive Statistics

\begin{tabular}{lrrrr}
\hline & Mean/Proportion & st.d. & Min & Max \\
\hline Depressive Symptoms & 9.61 & 6.10 & 1 & 43 \\
Friendship Network Structure & & & & \\
Network size & 8.93 & 4.42 & 1 & 34 \\
Alter-density & .21 & & 0 & 1 \\
Reciprocated school close friend & .45 & & 0 & 1 \\
Unreciprocated school close friend & .18 & & 0 & 1 \\
Non-school close friend & .24 & & 0 & 1 \\
No close friend & .12 & & 0 & 1 \\
Network Control Variables & & & & \\
\# Non-school friends & 2.14 & 2.21 & 0 & 10 \\
\# Missing school friend nomination & .25 & .57 & 0 & 5 \\
Social Perception & & & & \\
Perceived belonging & 9.49 & 2.55 & 1 & 13 \\
Perceived friend support & 4.28 & .77 & 1 & 5 \\
Demographic Characteristics & & & & \\
Female & .52 & & 0 & 1 \\
Grade & 9.35 & 1.62 & 6 & 12 \\
White & .75 & & 0 & 1 \\
Black & .17 & & 0 & 1 \\
Latino & .07 & & 0 & 1 \\
Household income (in \$1,000s) & 48.12 & 49.03 & 1 & 1000 \\
Missing on household income & .20 & & 0 & 1 \\
School size & 878.14 & 780.15 & 26 & 3334 \\
\# of years at current school & 2.84 & 1.61 & 1 & 6 \\
\# of years at current residence & 6.84 & 5.71 & 0 & 19 \\
\hline
\end{tabular}

Notes: Standard deviations are only reported for non-dummy variables. The count includes the focal adolescent.

$\mathrm{N}=9097$

could include romantic relationships, it is hard to speculate on why having friends outside of school would compromise the mental health of adolescent girls.

Models 2 and 3 test the hypothesis that small network size contributes to depressed mood even with the presence of one close same-sex friend. Model 2 shows the importance of having a close same-sex friend. Compared to those who do not have a same-sex close friend, having a reciprocated or non-reciprocated same-sex close friend in school is associated with lower depressive symptoms compared to adolescents without a same-sex close friend (-.880, $p<.01$ and $-.829, p<.01$, respectively). Having a same-sex close friend who does not attend one's school is also associated with lower depressive symptoms compared to adolescents 


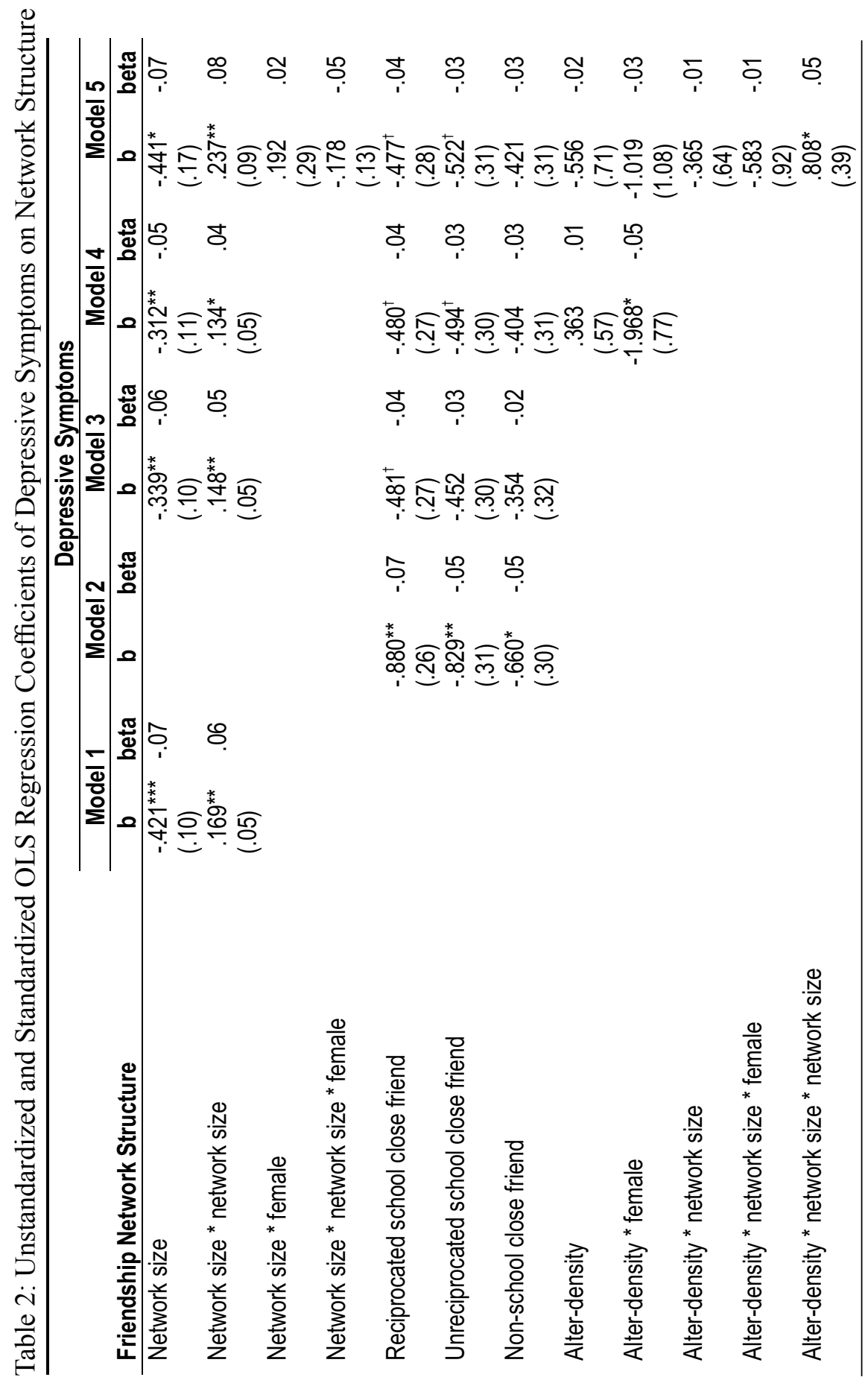




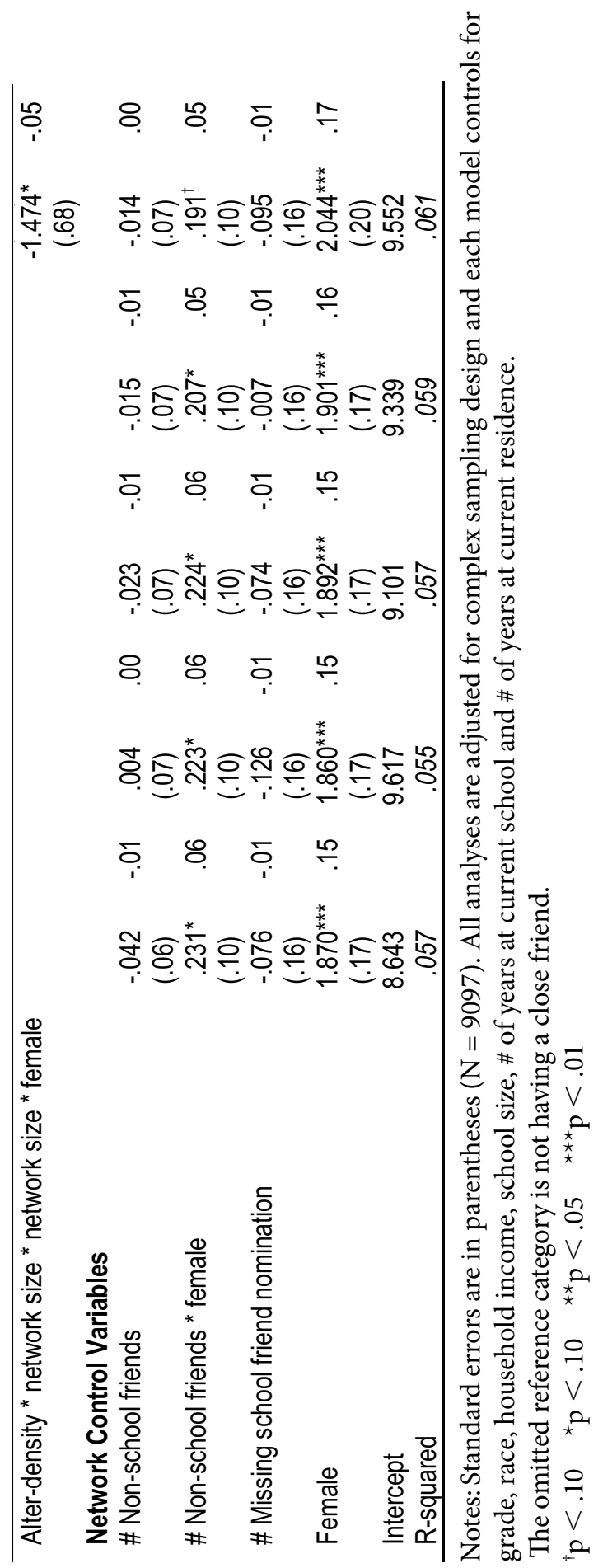


without a same-sex close friend (-.660, $p<.05)$. The consistent pattern of association across the close friendship variables indicates that having a close same-sex friend is protective, regardless of whether the friendship is reciprocated or whether the friend attends the same school. Model 3 shows that although having a close friend protects against depressive symptoms, it does not attenuate the association between network size and depressive symptoms. Thus, the effects of having a friend and network size appear to be additive. One friend is protective, but each additional friend is incrementally better, up to roughly 11 friends.

Model 4 shows the additive effects of network size and alter-density on depressive symptoms. The influence of alter-density on depressive symptoms varies by gender $(b=-1.968, p<.05)$. Among girls, having a higher proportion of friends who are also friends with one another is associated with lower levels of depressive symptoms. Alter-density does not have a significant effect on depressive symptoms among boys ( $b=$ $.363, n s)$. The final model in Table 2 investigates whether the negative effect of over-integration is exacerbated among adolescents in fragmented friendship networks (i.e., networks with low alter-density), and if this effect is stronger among girls. This hypothesis implies a four-way interaction between alter-density, the quadratic term for network size and gender. The four-way interaction explains additional variance in the model $(F=$ 7.53, $p<.01)$. To ease interpretation, Figure 2 visually displays the results from Model 5. The dashed lines show the predicted values for girls and the solid lines show the predicted values for boys. The lines marked with a diamond symbol represent adolescents with fragmented networks ( $\sim 10 \%$ alter density; the $25^{\text {th }}$ percentile for alter-density) and the unmarked lines represent adolescents with more cohesive network ( $\sim 30 \%$ alterdensity the $75^{\text {th }}$ percentile for alter-density). The total height of the graph represents two-thirds of a deviation for depressive symptoms.

For adolescent girls, large network size in conjunction with a fragmented social network is associated with the highest levels of depressive symptoms (dashed marked line). In contrast, high network cohesion protects girls in large networks from depressive symptoms (dashed unmarked line). Large network size is not associated with elevated levels of depressive symptoms for girls whose friends are friends with each other. For adolescent girls, there is no such thing as too many friends in a cohesive network, at least in terms of predicting depressive symptoms. Among girls who have 12 friends, there is about a one-fifth of standard deviation difference in depressive symptoms between girls who are in fragmented networks and those in cohesive networks. These higher levels of depressive symptoms occur among roughly 20 percent of adolescent girls in the sample who have networks with 12 or more friends. It is important to keep in mind that due to data limitations we underestimate 
Figure 2. Predicted Value of Depressive Symptoms across Values of Alter-Density and Degree by Gender



the true size of adolescent friendship networks. Furthermore, in this sample, the average size of a network is almost nine friends, which is where the divergence in depressive symptoms across values of alterdensity begins (see Figure 2).

The story is quite different for boys, represented by the solid lines in Figure 2. For adolescent boys, large network size in conjunction with a fragmented social network is associated with the lowest levels of depressive symptoms (solid marked line). This opposing trend compared to girls is clearly visible in Figure 2; compare the dashed marked line to the solid marked line. As network size increases, boys with fragmented networks and girls with cohesive networks experience declines in depressive symptoms. Among boys with cohesive networks (solid unmarked line), the association between network size and depressive symptoms is curvilinear. Having too few or too many friends is associated with elevated depressive symptoms. Adolescent boys in cohesive networks with roughly 10 friends report the lowest levels of depressive symptoms. The difference between adolescent boys with no friends and boys with 10 friends is about twofifths of a standard deviation for depressive symptoms.

In sum, over-integration is associated with higher levels of depressive symptoms among girls with fragmented networks and among boys with cohesive networks. In contrast, adolescent girls with large cohesive networks and boys with large fragmented networks tend to have the 
lowest levels of depressive symptoms. These findings, however, should be interpreted with a modicum of caution because Model 5 shows signs of multicollinearity. The standard errors for alter-density and network size between models 4 and 5 increase, and the VIF scores range 2-5 for the interaction terms in Model 5 . The randomly selected subset analyses and analyses stratified by gender and levels of alter-density do confirm the results of Model 5.

\section{Do Social Belonging and Peer Support Mediate the Effects of Under-Integration?}

The final hypothesis is that social belonging and friend support mediate the relationship between having few friends and depressive symptoms. For this to be true, belonging and support need to be related to both network size and depressive symptoms. Table 3 demonstrates the relationship of social belonging and friend support to network size. The relationship was expected to be linear; however, as shown in Table 3, non-linear associations are present. Including the squared term for network size in models 1 and 2 explains additional variation in both perceived belonging (Model $1, \mathrm{~F}=$ 29.47, $p<.001$ ) and perceived friend support (Model 2, $F=25.90, p<.001$ ). The nonlinear associations take the form of a diminishing returns effect. As network size increases, levels of perceived belonging and friend support also increase, but only to a certain point. For perceived belonging, the curve flattens out once the number of friends an adolescent has exceeds approximately 18 friends. For perceived friend support, the slope of the curve flattens out once the number of friends an adolescent has exceeds approximately 13 friends. Importantly, having a large network does not appear to compromise perceived belonging and friend support; rather, after a certain point there is no added benefit to having an additional friend.

The second requisite for a mediator is an association with the dependent variable. Model 1 in Table 4 shows that both social belonging and peer support are negatively related to depressive symptoms. Adolescents with higher levels of perceived belonging and support report fewer depressive symptoms. The remaining models in Table 4 test the hypothesis that perceived belonging and support mediate the association between having few friends and depressive symptoms. As stated previously, these variables are expected to mediate the ill effects of having few friends, but not the ill effects of too many friends. Model 2 shows that the curvilinear association between network size and depressive symptoms disappears when perceived belonging and support are included in the model. The squared term for network size does not explain additional variation in Model 2 ( $F=2.16$, ns). Model 3 drops the squared term to reveal a significant linear association between network size and depressive symptoms, controlling for perceived belonging and sup- 
Table 3: Unstandardized and Standardized OLS Regression Coefficients of Perceptions of Social Relationships on Network Structure

\begin{tabular}{|c|c|c|c|c|}
\hline \multirow[b]{2}{*}{ Friendship Network Structure } & \multicolumn{2}{|c|}{$\begin{array}{c}\text { Perceived } \\
\text { Belonging }\end{array}$} & \multicolumn{2}{|c|}{$\begin{array}{c}\begin{array}{c}\text { Perceived } \\
\text { Friend Support }\end{array} \\
\text { Model } 2 \\
\end{array}$} \\
\hline & b & beta & b & beta \\
\hline Network size & $\begin{array}{l}.100^{\star \star *} \\
(.02)\end{array}$ & .14 & $\begin{array}{l}.492^{\star * *} \\
(.06)\end{array}$ & .20 \\
\hline Network size * network size & $\begin{array}{l}-.025^{\star *} \\
(.01)\end{array}$ & -.07 & $\begin{array}{l}-.091^{* * *} \\
(.02)\end{array}$ & -.07 \\
\hline Reciprocated school close friend ${ }^{b}$ & $\begin{array}{l}.002 \\
(.04)\end{array}$ & .00 & $\begin{array}{l}.086 \\
(.15)\end{array}$ & .02 \\
\hline Unreciprocated school close friend ${ }^{b}$ & $\begin{array}{l}-.060 \\
(.05)\end{array}$ & -.03 & $\begin{array}{l}.210 \\
(.15)\end{array}$ & .03 \\
\hline Non-school close friend ${ }^{b}$ & $\begin{array}{l}-.040 \\
(.04)\end{array}$ & -.02 & $\begin{array}{l}-.010 \\
(.15)\end{array}$ & .00 \\
\hline Alter-density & $\begin{array}{l}.163^{* *} \\
(.06)\end{array}$ & .05 & $\begin{array}{l}.289 \\
(.19)\end{array}$ & .03 \\
\hline \multicolumn{5}{|l|}{ Network Control Variables } \\
\hline \# Non-s & $\begin{array}{l}.025^{\star *} \\
(.01)\end{array}$ & .07 & $\begin{array}{l}-.022 \\
(.03)\end{array}$ & -.02 \\
\hline \# Non-school friends * female & $\begin{array}{l}-.016 \\
(.01)\end{array}$ & -.03 & $\begin{array}{l}-.070^{\dagger} \\
(.04)\end{array}$ & -.04 \\
\hline \# Missing school friend nomination & $\begin{array}{l}.005 \\
(.02)\end{array}$ & .00 & $\begin{array}{l}.006 \\
(.07)\end{array}$ & .00 \\
\hline Female & $\begin{array}{l}.229^{* * *} \\
(.02)\end{array}$ & .15 & $\begin{array}{l}-.163^{\dagger} \\
(.10)\end{array}$ & -.03 \\
\hline Intercept & 3.820 & & 9.980 & \\
\hline R-squared & .071 & & .057 & \\
\hline
\end{tabular}

Notes: Standard errors are in parentheses all analyses are adjusted for complex sampling design and each model controls for grade, race, household income, school size, \# of years at current school and \# of years at current residence $(\mathrm{N}=9097)$. The omitted reference category is not having a school close friend ${ }^{\dagger} \mathrm{p}<.10 \quad{ }^{*} \mathrm{p}<.10 \quad{ }^{* *} \mathrm{p}<.05 \quad{ }^{* * *} \mathrm{p}<.01$

port. The association is small but positive $(b=.187, p<.05)$; adolescents with larger networks report more depressive symptoms. As expected, perceived belonging and support mediate the ill effects of small friendship networks but not large ones, in which depressive symptoms remain elevated.

The higher levels of depressive symptoms among adolescents with many friends cannot be explained by the extent to which they perceive belonging at school or perceive support from their friends. Furthermore, in Model 4 of Table 4, the four-way interaction still explains additional 
variation in the model, over and above the effect of belonging and support $(F=8.64, p<.01)$. The differential effects of over-integration across gender and levels of network cohesion do not disappear upon controlling for perceived belonging at school or perceived support. Adolescent girls in large fragmented networks report higher levels of depressive symptoms compared to girls with large cohesive networks, whereas adolescent boys with large cohesive networks report the highest levels of depressive symptoms compared to boys with large fragmented networks.

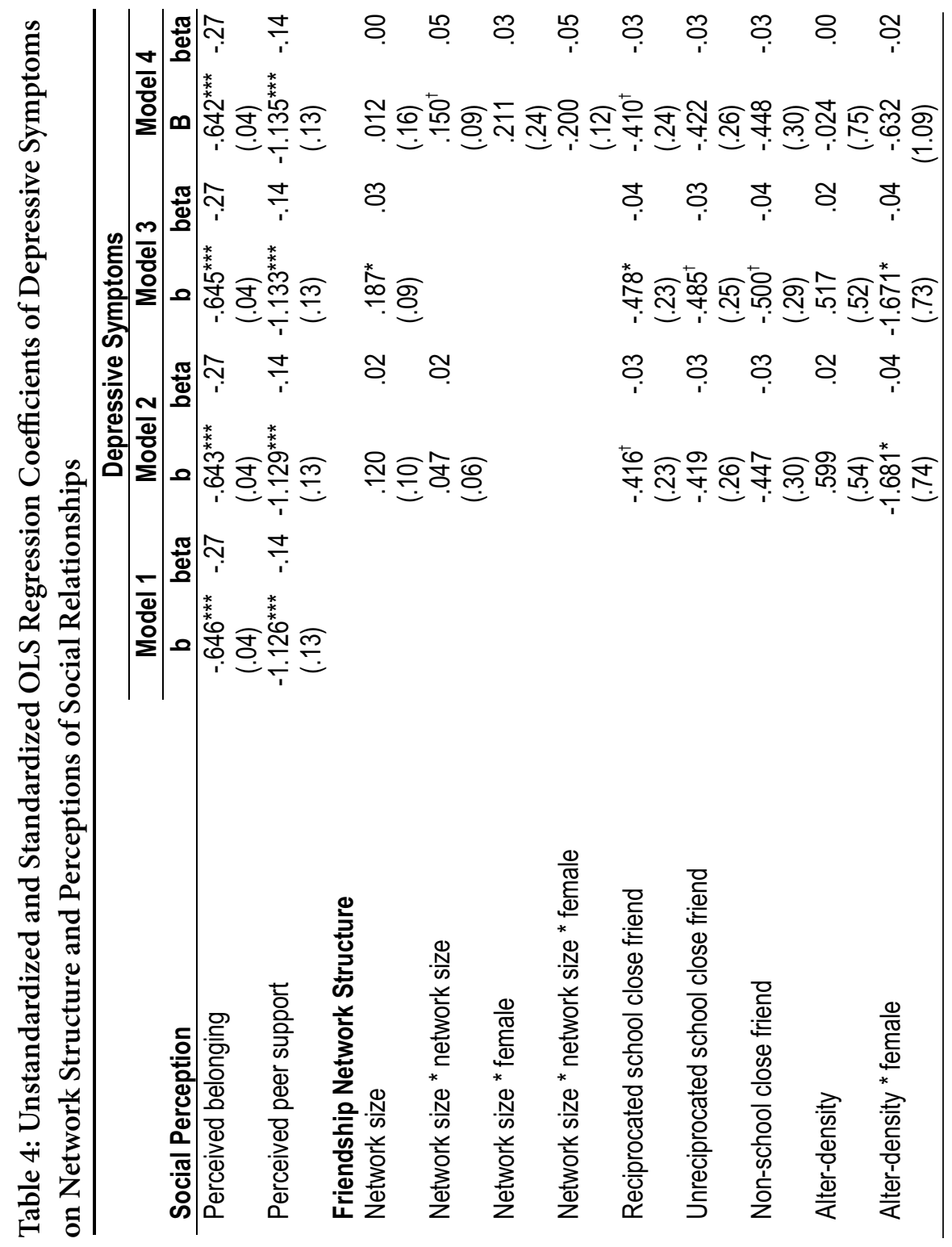




\section{Discussion and Conclusion}

This article reexamined the association between social integration and mental health. The association between social integration on depressive symptoms is curvilinear. Consistent with much previous research, underintegration (i.e., having too few friends) is associated with higher levels of depressive symptoms (Brendgen et al. 2000; Ueno 2005). Furthermore, under-integration is associated with elevated depressive symptoms,

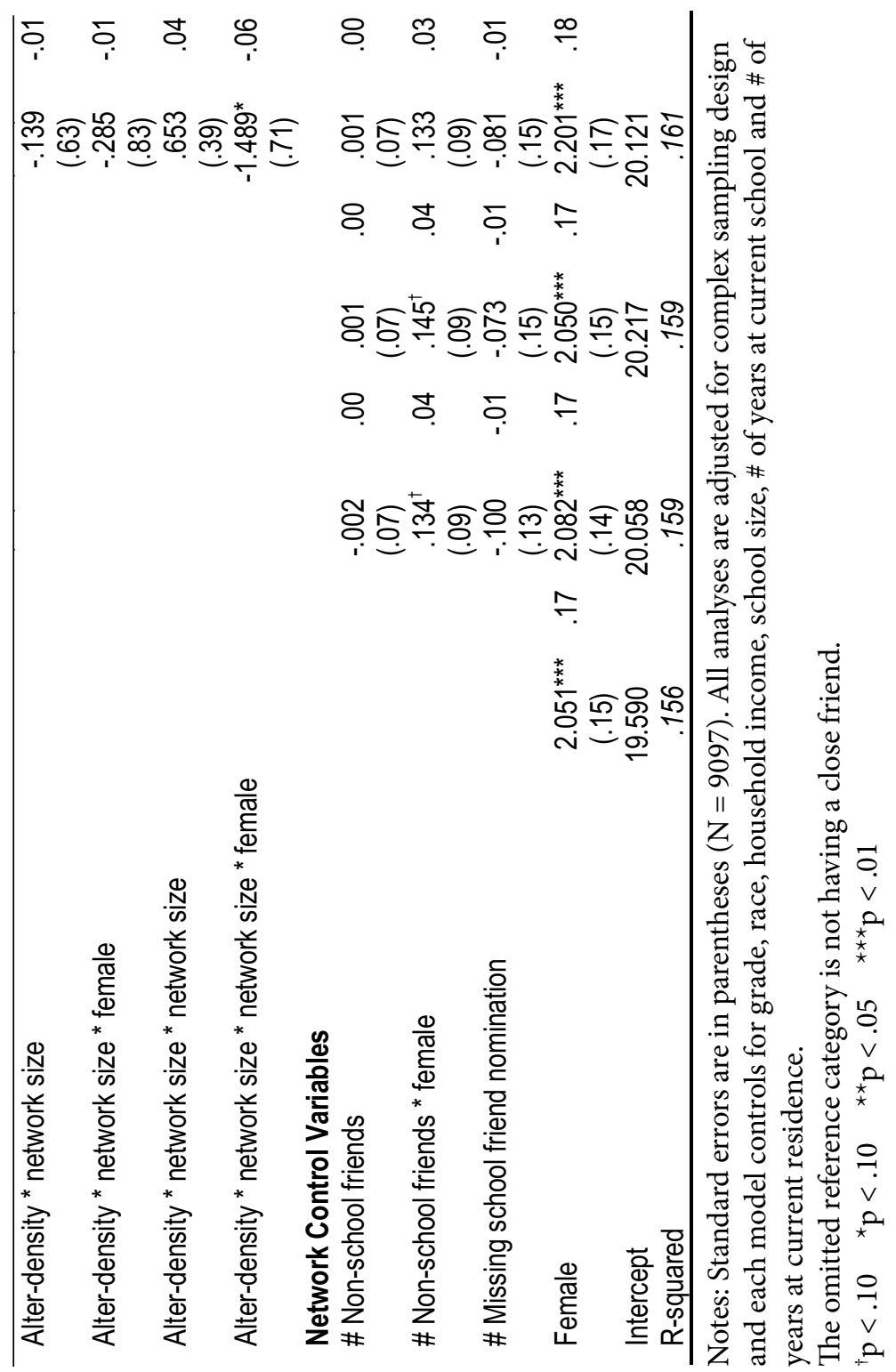


regardless of the presence of a close friend. Adolescents need multiple friendships to meet their relationship needs (Crosnoe and Needham 2004; Crosnoe and McNeely forthcoming). Having one close friend is not enough to ward off the ill effects of under-integration. As predicted, underintegration is equally bad for boys and girls. This finding is consistent with qualitative evidence that adolescent boys desire close relations with peers as much as girls desire close relations (Chu 2005).

This is the first study to our knowledge to test the hypothesis that over-integration increases depressive symptoms and to provide empirical evidence in support of the theoretical claim that having too many friends may compromise mental health. Over-integration is likely to lead to higher levels of depressive symptoms, due to higher levels of role strain placed on adolescents attempting to meet the obligations of numerous friends. It is, however, important to contextualize over-integration because its effects on depressive symptoms vary as a function of gender and network cohesion. Among adolescents girls, over-integration is associated with higher levels of depressive symptoms only when networks are fragmented (i.e., few of an adolescent's friends are friends with each other). In contrast, highly cohesive networks protect against developing depressive symptoms among girls in over-integrated networks. Among girls, social networks can be large as long as the adolescent's friends tend to be friends with one another. For boys, over-integration is associated with an increase in depressive symptoms when network cohesion is high. In contrast to adolescent girls, adolescent boys in large fragmented networks report the lowest levels of depressive symptoms. For boys, low levels of network cohesion protect against the potential ill effects of over-integration.

These findings have important implications. First, researchers traditionally focus on studying adolescents with few social ties. However, we cannot assume that teens with a lot of friends, and who may be quite social, are not experiencing depressive symptoms. Second, adolescents experience social networks holistically. Breaking down each particular network characteristics into a set of additive, independent variables may not accurately capture the influence of network characteristics on adolescent health. Theoretically grounded hypotheses about how a constellation of network characteristics jointly influence adolescent health will help advance our understanding in this nascent line of research. Third, the gathering of full rank network data will also be important for advancing this field of research. Within this research, it was the social ties of an adolescent's friends (network cohesion) that provided the most insight into the curvilinear association between network size and depressive symptoms in adolescence.

Can perceptions of belonging and support from friends mediate the effects of under- and over-integration on depressive symptoms? The answer is yes and no. The perception of belonging and support explained 
one end of the social integration continuum, but not the other. Adolescents with few or no friends (i.e., under-integrated) reported lower levels of perceived support and belonging; and these perceptions mediated the association between under-integration and depressive symptoms. For the most part, having many friends (i.e., over-integration) does not compromise positive perceptions of support and belonging. As a result, perceptions of social relationships did not mediate the ill effects of over-integration on depressive symptoms.

The findings from this research should be considered within the limitations of the research. First, the measure of network size is truncated due to the 10 friend nomination limit; thereby, underestimating both the size and cohesiveness of an adolescent's network, especially among adolescents with larger networks. Despite this underestimation, a curvilinear effect of network size on depressive symptoms is found. Second, the complexity of the statistical models, in particular the four-way interaction, raises the possibility of multicollinearity problems. For these reasons, the findings from this research should be considered preliminary until future research can replicate these results. The models with two-way interaction terms appear not to suffer from multicollinearity; therefore, we are confident that the association between network size and depressive symptoms is curvilinear and that network cohesion provides more protection against depressive symptoms for girls than boys. Finally, network structure is assumed to be causally linked to perceptions of social relationships and depressive symptoms. It is possible that depressed adolescents are inclined to socially isolate themselves from or be isolated by other students at school (Link et al. 1989). With regard to over-integration, however, it is less likely that depressed adolescents will select themselves into over-integrated networks.

In spite of these limitations, this research adds to the limited number of studies on the association between social network structure and mental health by providing empirical evidence for the often theorized ill effects of over-integration on depressive symptoms (Durkheim 1951; Pescosolido and Levy 2002). Furthermore, the importantance of investigating a constellation of network characteristics, such as interactions between social integration and network cohesion, is shown. We also demonstrate that a similar constellations of network characteristics can be experienced in dramatically different ways by boys and girls and, as a result, foster different developmental outcomes.

\section{Notes}

1. Ueno (2005) did graph mean differences in depressive symptoms across network size and found a linear trend. Although our study uses the same data as Ueno (2005), our measure of network size differs and we engage in a more rigorous empirical test of the potentially ill effects of over-integration. 
Ueno (2005) measured network size with the number of sent-friendship nominations. This measure relies solely on self-reports and is capped at 10 possible nominations. Respondents in Add Health were allowed to nominate five friends of each gender for a maximum of 10 friends. Our measure of network size draws on information from both the number of friendship nominations made and the number received from other students in the school. Our measure overcomes the limitations of self-report data and surpasses the artificial ceiling of a network size of 10 friends. We believe incorporating information from both sent and received friendship nominations more accurately assesses the size of an adolescent's friendship network, especially large networks. Over-integrated adolescents might have listed more friends if they were given the opportunity to do so.

\section{References}

Anderson, Robert. 2001. "Deaths: Leading Causes for 1999." National Vital Statistics Report; Volume 49, Number 11. Hyattsville, MD: National Center for Health Statistics.

Baumeister, Roy F., and Mark R. Leary. 1995. "The Need to Belong: Desire for Interpersonal Attachments As a Fundamental Human Motivation." Psychological Bulletin 117(3):497-529.

Beam, Margaret R., Virginia Gil-Rivas, Ellen Greenberger and Chuansheng Chen. 2002. "Adolescent Problem Behavior and Depressed Mood: Risk and Protection Within and Across Social Contexts." Journal of Youth and Adolescence 31(5):343-57.

Belsher, Gayle, and Charles G. Costello. 1988. "Relapse After Recovery From Unipolar Depression: a Critical Review." Psychological Bulletin 104(1):84-96.

Bollen, Kenneth A., and Rick H. Hoyle. 1990. "Perceived Cohesion: A Conceptual and Empirical Examination." Social Forces 69(2):479-504.

Brendgen, Mara, Frank Vitaro and William M. Bukowski. 2000. "Deviant Friends and Early Adolescents' Emotional and Behavioral Adjustment." Journal of Research on Adolescence 10(2):173-89.

Burt, Ronald.S. 1998. "The Gender of Social Capital." Rationality and Society 10(1):5-46.

Chantala, Kim. 2006. Guidelines for Analyzing Add Health. Chapel Hill, NC: Carolina Population Center.

Chu, Judy Y. 2005. "Adolescent Boys' Friendships and Peer Group Culture." New Directions for Child and Adolescent Development 2005(107):7-22.

Crosnoe, Robert, and Clea McNeely. 2008. "Peer Relations, Adolescent Behavior, and Public Health Research and Practice." Family and Community Health 31(Supplement 1):S71-S80. 
Crosnoe, Robert, and Belinda Needham. 2004. "Holism, Contextual Variability, and the Study of Friendships in Adolescent Development." Child Development 75(1):264-79.

Durkheim, Emile. 1951. Suicide. Free Press.

Eccles, Jacquelynne S., Carol Midgley, Allan Wigfield, Christy Miller Buchanan, David Reuman, Constance Flanagan and Douglas Mac Iver. 1993. "Development During Adolescence: The Impact of Stage-Environment Fit on Young Adolescents' Experiences in Schools and in Families." American Psychologist 48(2):90-101.

Echambadi, Raj, and James D. Hess. 2007. "Mean-Centering Does Not Alleviate Collinearity Problems in Moderated Multiple Regression Models." Marketing Science 26(3):438-45.

Eder, Donna. 1985. "The Cycle of Popularity: Interpersonal Relations Among Female Adolescents." Sociology of Education 58(3):154-65.

Eder, Donna, and Janet L. Enke. 1991. "The Structure of Gossip: Opportunities and Constraints on Collective Expression Among Adolescents." American Sociological Review 56(4):494-508.

Eder, Donna, Catherine C. Evans and Stephen Parker. 1995. School Talk: Gender and Adolescent Culture. Rutgers University Press.

Ennett, Susan T., Karl E. Bauman, Andrea Hussong, Robert Faris, Vangie A. Foshee and Li Cai. 2006. "The Peer Context of Adolescent Substance Use: Findings From Social Network Analysis." Journal of Research on Adolescence 16(2):159-86.

Fagot, Beverly I. 1985. "Beyond the Reinforcement Principle: Another Step Toward Understanding Sex Role Development." Developmental Psychology 21(6):1097-104.

Formoso, Diana, Nancy A. Gonzales and Leona S. Aiken. 2000. "Family Conflict and Children's Internalizing \& Externalizing Behavior: Protective Factors." American Journal of Community Psychology 28(2):175-99.

Friedkin, Noah E. 2004. "Social Cohesion." Annual Review of Sociology 30:409-25. . 1984. "Structural Cohesion and Equivalence Explanations of Social Homogeneity." Sociological Methods and Research 12(3):235-61.

Frydenberg, Erica, and Lewis Ramon. 1993. "Boys Play Sport and Girls Turn to Others: Age, Gender and Ethnicity As Determinants of Coping." Journal of Adolescence 16(3):253-66.

Furman, Wyndol, and Duane Buhrmester. 1992. "Age and Sex Differences in Perceptions of Networks of Personal Relationships." Child Development 63(1):103-15. 
Gecas, Victor. 1986. "The Motivational Significance of Self-Concept for Socialization Theory." Pp. 131-56. Advances in Group Processes: Theory and Research, Volume 3. E.J. Lawler, editor. JAI Press.

Gifford-Smith, Mary E., and Celia A. Brownell. 2003. "Childhood Peer Relationships: Social Acceptance, Friendships, and Peer Networks." Journal of School Psychology 41(4):235-84.

Greene, A.L. 1988. "Early Adolescents' Perceptions of Stress." The Journal of Early Adolescence 8(4):391-403.

Haines, Valerie A., John J. Beggs and Jeanne S. Hurlbert. 2002. "Exploring the Structural Contexts of the Support Processes: Social Networks, Social Statuses, Social Support, and Psychological Distress." Pp. 269-92. Social Networks and Health. Judith A. Levy and Bernice A. Pescosolido, editors. JAI Press.

Hair, Joseph F., Ronald L. Tatham, Rolph Anderson and Wiliam C. Black. 2006. Multivariate Data Analysis. Prentice Hall.

Hansell, Stephen. 1985. "Adolescent Friendship Networks and Distress in School." Social Forces 63(3):698-715.

Haynie, Dana L. 2001. "Delinquent Peers Revisited: Does Network Structure Matter?" American Journal of Sociology 106(4):1013-57.

Haynie, Dana L., and D.W. Osgood. 2005. "Reconsidering Peers and Delinquency: How Do Peers Matter?" Social Forces 84(2):1109-30.

House, James S., Debra Umberson and Karl R. Landis. 1988. "Structures and Processes of Social Support." Annual Review of Sociology 14:293-318.

Ibarra, H. 1997. "Paving an Alternative Route: Gender Differences in Managerial Networks." Social Psychology Quarterly 60(1):91-102.

Jaccard, James, Choi K. Wan and Robert Turrisi. 1990. "The Detection and Interpretation of Interaction Effects Between Continuous Variables in Multiple Regression." Multivariate Behavioral Research 25(4):467-78.

Joyner, Kara, and J. Richard Udry. 2000. "You Don't Bring Me Anything but Down: Adolescent Romance and Depression." Journal of Health and Social Behavior 41(4):369-91.

Kandel, Denise, Victoria Raveis and Mark Davies. 1991. "Suicidal Ideation in Adolescence: Depression and, Substance Use and Other Risk Factors." Journal of Youth and Adolescence 20(2):289-309.

Kossinets, Gueorgi. 2006. "Effects of Missing Data in Social Networks." Social Networks 28(3):247-68. 
Kovacs, Maria, Terry Feinberg, Mary Ann Crouse-Novak, Stana Paulauskas and Richard Finkelstein. 1984. "Depressive Disorders in Childhood: A Longitudinal Prospective Study of Characteristics and Recovery." Archives of General Psychiatry 41(3):219-39.

Kromrey, Jeffrey D., and Lynn Foster-Johnson. 1998. "Mean Centering in Moderated Multiple Regression: Much Ado About Nothing." Educational and Psychological Measurement 58(1):42-67.

Laible, Deborah J., Gustavo Carlo and Marcela Raffaelli. 2000. "The Differential Relations of Parent and Peer Attachment to Adolescent Adjustment." Journal of Youth and Adolescence 29(1):45-59.

Link, Bruce G., F.T. Cullen, E.L. Struening, P.E. Shrout and B.P. Dohrenwend. 1989. "A Modified Labeling Theory Approach to Mental Disorders: an Empirical Assessment." American Sociological Review 54(3):400-23.

Marcotte, Diane, Michel Alain and Marie-Josee Gosselin. 1999. "Gender Differences in Adolescent Depression: Gender-Typed Characteristics or Problem-Solving Skills Deficits?" Sex Roles 41(112):31-48.

Marcussen, Kristen, Christian Ritter and Deborah J. Safron. 2004. "The Role of Identity Salience and Commitment in the Stress Process." Sociological Perspectives 47(3):289-312.

McNeely, Clea, and Christina Falci. 2004. "School Connectedness and the Transition Into and Out of Health-Risk Behavior Among Adolescents: A Comparison of Social Belonging and Teacher Support." Journal of School Health 74(7):284-92.

Messerschmidt, James W. 2000. Nine Lives: Adolescent Masculinities, the Body, and Violence. Westview Press.

Moody, James. 2001a. "Race, School Integration, and Friendship Segregation in America." American Journal of Sociology 107(3):679-716.

2001b. Using the Social Network Data From Add Health. Bethesda, Maryland: Add Health Users Workshop.

Nada-Raja, Shyamala, Rob McGee and Warren Stanton. 1992. "Perceived Attachments to Parents and Peers and Psychological Well-Being in Adolescence." Journal of Youth and Adolescence 21(4):471-85.

Osgood, Wayne, Barbara J. McMorris and Maria T. Potenza. 2002. "Analyzing Multiple-Item Measures of Crime and Deviance I: Item Response Theory Scaling." Journal of Quantitative Criminology 18(4):267-96.

Pearlin, Leonard I. 1983. "Role Strains and Personal Stress." Pp. 3-32. Psychosocial Stress: Trends in Theory and Research. Howard B. Kaplan, editor. Academic Press. 
Pescosolido, Bernice A., and Judith A. Levy. 2002. "The Role of Social Networks in Health , Illness, Disease and Healing." Pp. 3-25. Social Networks and Health, Volume 8. Judith Levy and Bernice Pescosolido, editors. Elsevier Science.

Raghunathan, T.E., Peter W. Solenberger and John Van Hoewyk. 2002. IVEware: Imputation and Variance Estimation Software User Guide. University of Michigan: Survey Research Center, Institute for Social Research.

Rosenberg, Morris, and B.C. Cullough. 1981. "Mattering: Inferred Significance and Mental Health Among Adolescents." Research in Community and Mental Health 2:163-82.

Rosenfield, Sarah, Mary C. Lennon and Helene R. White. 2005. "The Self and Mental Health: Self-Salience and the Emergence of Internalizing and Externalizing Problems." Journal of Health and Social Behavior 46(4):323-40.

Rushton, Jerry, Michelle Forcier and Robin Schectman. 2002. "Epidemiology of Depressive Symptoms in the National Longitudinal Study of Adolescent Health." Journal of the American Academy of Child and Adolescent Psychiatry 41(2):199-205.

Samejima, Fumiko 1969. "Estimation of Latent Ability Using a Response Pattern of Graded Scores." Psychometrika Monograph Supplement No.1734(4, Part 2):104-14.

Thoits, Peggy A. 1991. "On Merging Identity Theory and Stress Research." Social Psychology Quarterly 54(2):101-12.

Turner, R.Jay, and J.Blake Turner. 1999. "Social Integration and Support." Pp. 301 20. Handbook of the Sociology on Mental Health. Carol Aneshensel and Jo Phelan, editors. Kluwer Academic/Plenum Publishers.

Udry, J.Richard 2003. The National Longitudinal Study of Adolescent Health (Add Health), Waves I \& II, 1994-1996; Wave III, 2001-2002. Available at: http:// www.cpc.unc.edu/projects/addhealth/design.

Ueno, Koji. 2005. "The Effects of Friendship Networks on Adolescent Depressive Symptoms." Social Science Research 34(3):484-510.

Walker, John M., Paul M. Wasserman and Barry Wellman. 1993. "Statistical Models for Social Support Networks." Sociological Methods and Research 22(1):71-98.

Wasserman, Stanley, and Katherine Faust. 1994. Social Networks Analysis: Methods and Application. Cambridge University Press.

Wellman, Barry. 1988. "Structural Analysis: From Method and Metaphor to Theory and Substance." Pp. 19-61. Social Structures: A Network Approach. Barry Wellman and S. D. Berkowitz, editors. Cambridge University Press.

Zucker, Kenneth, Janice Kurita Anita Stern and Debra Wilson-Smith. 1995. "Children's Appraisals of Sex-Typed Behavior in Their Peers." Sex Roles 33(11):703-25. 
Appendix A: Average Network Size across Values of Alter-Density

\section{Network Size}

\begin{tabular}{lrcccc} 
Alter-Density \% & Mean & st.d. & Min & Max & \multicolumn{1}{c}{$N$} \\
\hline 0 & 3.51 & 2.03 & 1 & 13 & 1706 \\
$>0$ and $<20$ & 10.61 & 3.96 & 5 & 34 & 3638 \\
$\geqslant 20$ and $<40$ & 9.64 & 3.82 & 4 & 29 & 2650 \\
$\geqslant 40$ and $<60$ & 8.60 & 2.98 & 5 & 21 & 661 \\
$\geqslant 60$ & 5.47 & 2.68 & 3 & 17 & 442 \\
\hline
\end{tabular}

Note: $\mathrm{N}=9097$ 
32 - Social Forces 87(4) 